\title{
Desmoplastic Small Round Cell Tumors in a Young Man
}

\author{
Genryu Hirano ${ }^{1}$, Makoto Irie ${ }^{1}$, Yuta Nakashima ${ }^{2}$, Satoshi Shakado ${ }^{1}$, Tetsuro Sohda ${ }^{1}$, \\ Toshihiro Tanaka ${ }^{2}$, Yasushi Takamatsu ${ }^{2}$, Mikiko Aoki ${ }^{3}$, Kazuo Tamura $^{2}$, \\ Kazuki Nabeshima ${ }^{3}$ and Shotaro Sakisaka ${ }^{1}$
}

\begin{abstract}
A previously healthy 18-year-old man was admitted to our hospital with abdominal pain in September 2010. We performed a percutaneous biopsy of multiple intrahepatic masses. A diagnosis of desmoplastic small round cell tumors was confirmed based on the presence of a polyphenotypic immunoprofile (positivity for EMA, vimentin, cytokeratin, desmin and WT1) and characteristic EWS-WT1 gene fusion. Because the mass had invaded the mesentery and the disease had disseminated to liver, the patient received palliative chemotherapy with carboplatin, paclitaxel, vincristine, doxorubicin, cyclophosphamide, ifosfamide, etoposide and irinotecan. The maximal response to the chemotherapy was a partial remission. The patient died 20 months after diagnosis.
\end{abstract}

Key words: chemotherapy, desmoplastic small round cell tumor, EWS-WT1 gene fusion transcript, reverse transcriptase polymerase chain reaction

(Intern Med 52: 1909-1914, 2013)

(DOI: 10.2169/internalmedicine.52.0431)

\section{Introduction}

Desmoplastic small round cell tumor (DSRCT) is a rare and highly aggressive mesenchymal tumor that was first described as a separate identity in 1989 (1). Characterized by a predilection for young men, it typically presents as an intra-abdominal mass with multiple intra-peritoneal implants $(2,3)$. However, the site of origin may not be readily apparent (4). The diagnosis of DSRCT is established based on histology, immunochemistry and cytogenetics. Histological examinations reveal sheets or nests of uniform small, round or ovoid cells within a desmoplastic stroma $(5,6)$. DSRCT has a polyphenotypic immunoprofile and is positive for the EWS-WT1 fusion transcript $(7,8)$. Most patients are diagnosed in the advanced stage and have a poor prognosis. Despite the administration of intensive therapy, including surgery, radiotherapy and chemotherapy, the 5-year survival rate continues to be less than $15 \%(9-12)$. We herein report the case of a young man who was diagnosed with DSRCT and treated with combination chemotherapy.

\section{Case Report}

A previously healthy 18 -year-old man was admitted to our hospital with upper abdominal pain in September 2010. He had a palpable mass measuring approximately $4.0 \mathrm{~cm}$ in diameter in the middle of the lower abdominal region. His liver was palpable $7 \mathrm{~cm}$ below the right costal margin. The results of liver function tests showed elevated levels of transaminases, alkaline phosphatase (ALP) and gammaglutamyl transpeptidase (GGT). The serum neuron specific enolase level was $46.0 \mathrm{ng} / \mathrm{mL}$ (normal range, $0-10$ ) (Table). Ultrasonography showed multiple hypoechoic masses in the liver and a mass including spotty areas of calcification measuring $4.0 \mathrm{~cm}$ in diameter in the middle of the lower ab-

\footnotetext{
${ }^{1}$ Department of Gastroenterology and Medicine, Fukuoka University Faculty of Medicine, Japan, ${ }^{2}$ Department of Medical Oncology, Hematology and Infectious Disease, Fukuoka University Faculty of Medicine, Japan and ${ }^{3}$ Department of Pathology, Fukuoka University Faculty of Medicine, Japan

Received for publication February 22, 2013; Accepted for publication April 30, 2013

Correspondence to Dr. Genryu Hirano, g.hirano@minf.med.fukuoka-u.ac.jp
} 
dominal region (Fig. 1). Abdominal computed tomography (CT) demonstrated the presence of a lower abdominal mass measuring $4.0 \mathrm{~cm}$, hepatomegaly and multiple intrahepatic metastases. The lower abdominal mass exhibited less enhanced areas with spotty calcification (Fig. 2). Hybrid $\left[{ }^{18} \mathrm{~F}\right]$ fluorodeoxyglucose-positron emission tomography $\left({ }^{18} \mathrm{FDG}-\right.$ $\mathrm{PET} / \mathrm{CT}$ ) revealed multiple intrahepatic masses exhibiting accumulation of FDG (standardized uptake value (SUV) = 14.6, max) and a lower abdominal mass exhibiting accumulation of FDG (SUV =6.60) (Fig. 3). Upper gastrointestinal endoscopy, double-balloon endoscopy and colonoscopy demonstrated no localized lesions. The histopathologic results of the biopsy of the intrahepatic masses showed proliferation of atypical cells with hyperchromatic nuclei forming irregularly shaped small nests accompanied by fibromyxoid stroma (Fig. 4). The tumor cells coexpressed an epithelial membrane antigen (EMA) (Dako, Glostrup, Denmark), a mesenchymal marker (vimentin) (Dako) and a neuronal marker (neuron specific enolase) (Dako). Other immunohistochemistry findings showed that the tumor cells were positive for cytokeratins (CAM5.2, AE1/AE3) (Becton Dickinson, San Jose, CA, USA) and WT1 (Santa Cruz Biotechnology, Heidelberg, Germany) and negative for c-kit (IBL, Fujioka, Japan), CD19 (Dako), CD30 (Dako) and CD34 (Im-

Table. Laboratory Data on Admission

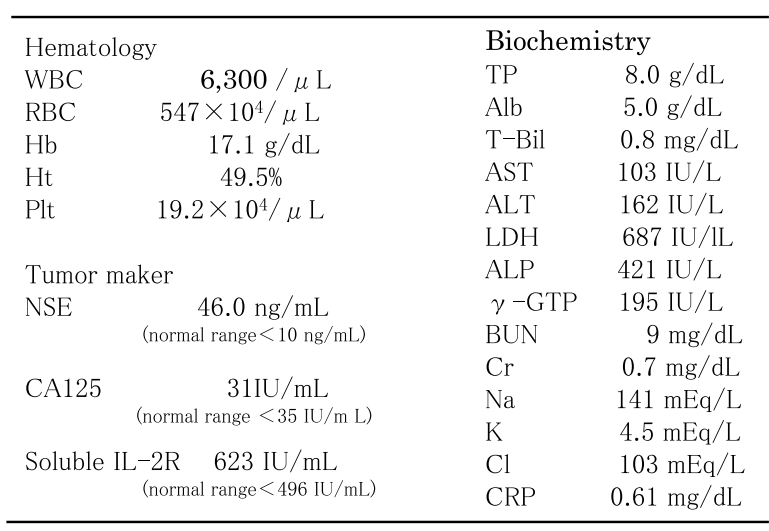
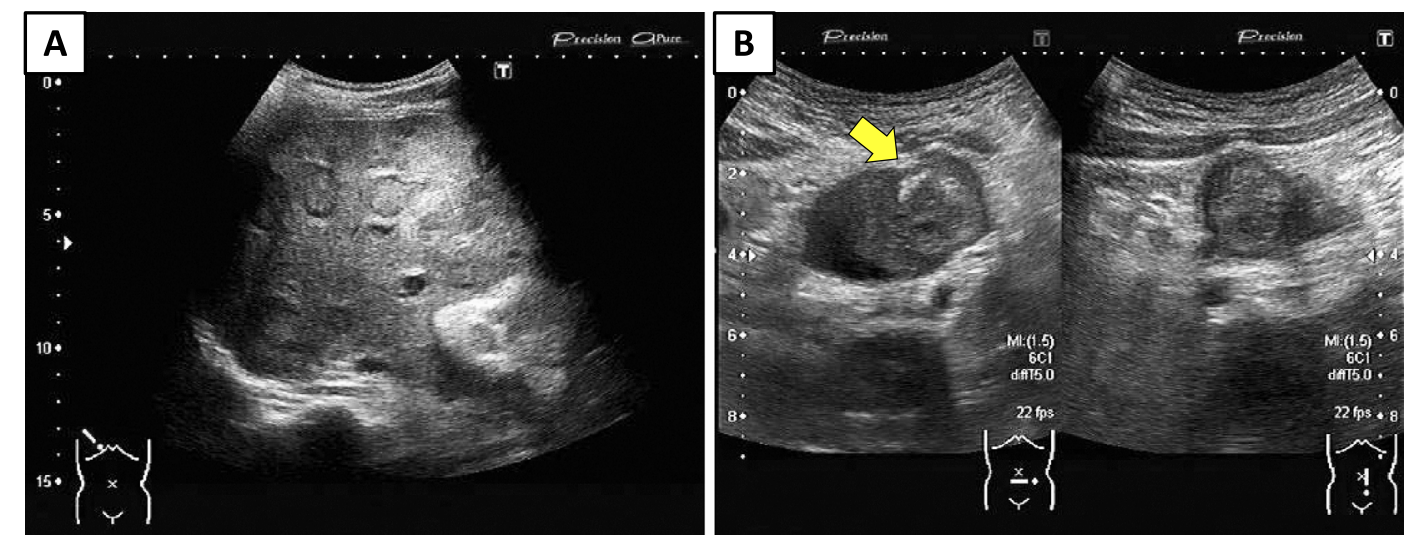

Figure 1. (A) Ultrasonography showed multiple hypoechoic masses in the liver. (B) A mass including an area of spotty calcification measuring $4.0 \mathrm{~cm}$ in diameter in the middle of the lower abdominal region (arrow). munotech, Marseille, France). Because the extraction of the messenger RNA was poor, the results of reverse transcriptase polymerase chain reaction (RT-PCR) for the EWS-WT1 gene fusion transcript were not confirmed. The characteristic reciprocal translocation that is specific to DSRCT was identified on fluorescence in situ hybridization (FISH) (Fig. 5A). Although the finding of positive cytokeratins were compatible with a diagnosis of epithelial tumors, a diagnosis of desmoplastic small round cell tumors was made due to the typical histological findings and karyotypic alterations. The patient was treated with chemotherapy consisting of carboplatin (AUC 6) plus paclitaxel $\left(200 \mathrm{mg} / \mathrm{m}^{2}\right.$ ) (CBDCA/ PTX). Six courses of chemotherapy were administered, and the tumor size was significantly. He achieved partial remission according to the RECIST criteria (13) (Response Evaluation Criteria in Solid Tumors). His general condition recovered and was sustained for two months. However, after six months, the patient again developed an increased level of neuron-specific enolase (NSE) and enlarged tumor size. He underwent a second liver biopsy to evaluate the histological changes, leading to successful detection of the EWS-WT1 fusion transcript on RT-PCR, which revealed a fusion product in EWS with exon 8 of WT1 and exon 9 of WT1 (Fig. 5B). The above molecular studies confirmed the diagnosis of desmoplastic small round cell tumors. The patient was then treated with a second-line chemotherapy P6 protocol consisting of seven courses of chemotherapy with cyclophosphamide $\left(2,100 \mathrm{mg} / \mathrm{m}^{2}\right)$, doxorubicin $\left(25 \mathrm{mg} / \mathrm{m}^{2}\right)$ and vincristine $\left(0.67 \mathrm{mg} / \mathrm{m}^{2}\right)$ (HD-CAV) alternating with ifosfamide $\left(3,000 \mathrm{mg} / \mathrm{m}^{2}\right)$ and etoposide $\left(250 \mathrm{mg} / \mathrm{m}^{2}\right)$. He responded to this regimen only transiently, and soon after the seven courses of second-line therapy, progressive disease was noted. We administered salvage chemotherapy with irinotecan (CPT-11 at $250 \mathrm{mg} / \mathrm{m}^{2}$ ). The lower abdominal tumor appeared to be stabilized. However, the liver tumors progressed. We decided not to continue with further treatment due to the rapid deterioration of the patient's condition. He died 20 months after receiving the initial diagnosis (Fig. 6). An autopsy was not performed. 

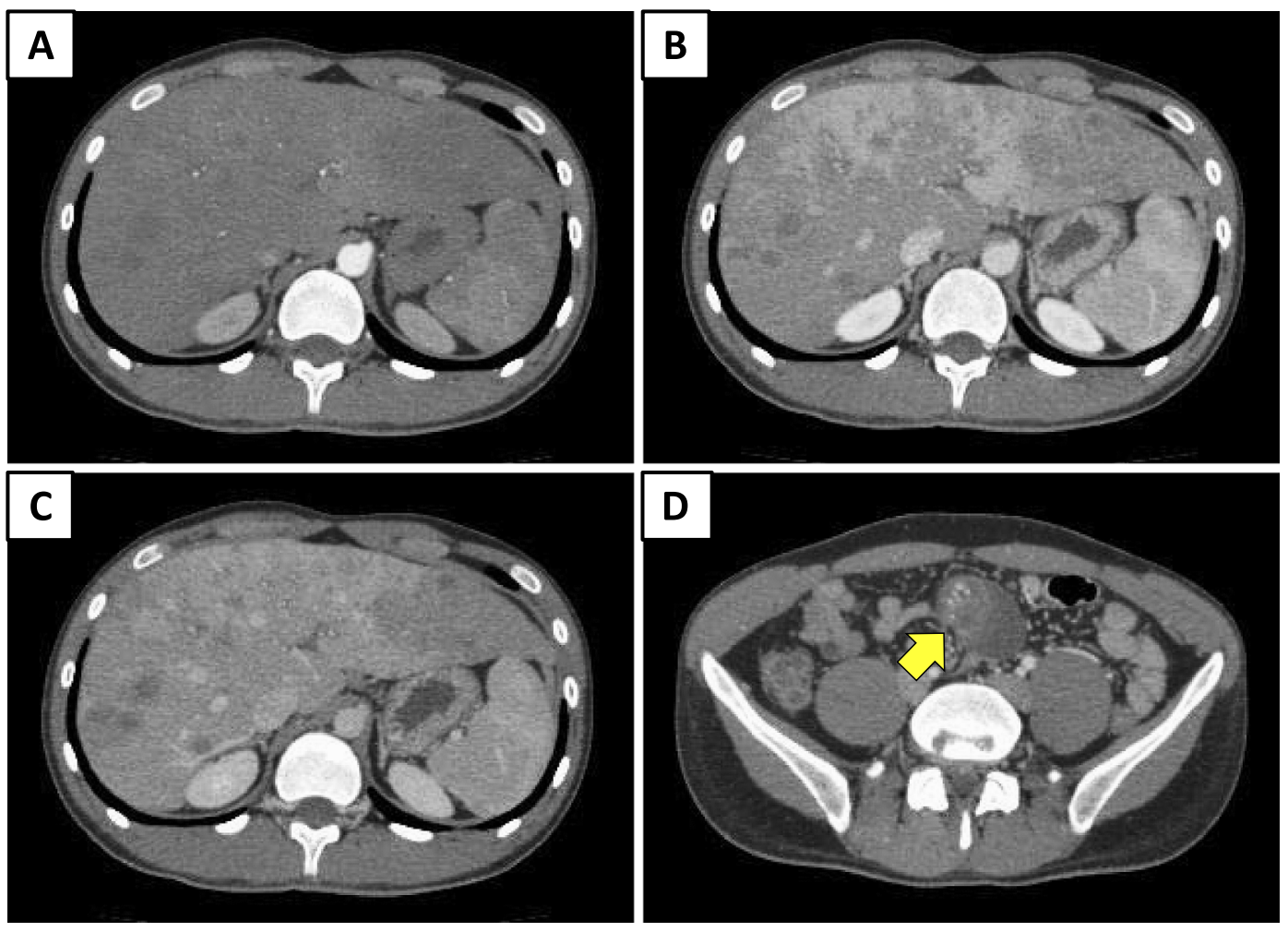

Figure 2. (A)-(C) Triple phase helical dynamic CT of the liver revealed the presence of hepatomegaly and multiple intrahepatic tumors. (A) Arterial phase CT showed heterogeneous masses in the liver. (B)(C) Portal venous phase and delayed phase CT showed heterogeneous hypoattenuating masses in the liver. (D) A lower abdominal mass measuring $4.0 \mathrm{~cm}$ exhibited less enhanced areas of spotty calcification (arrow).
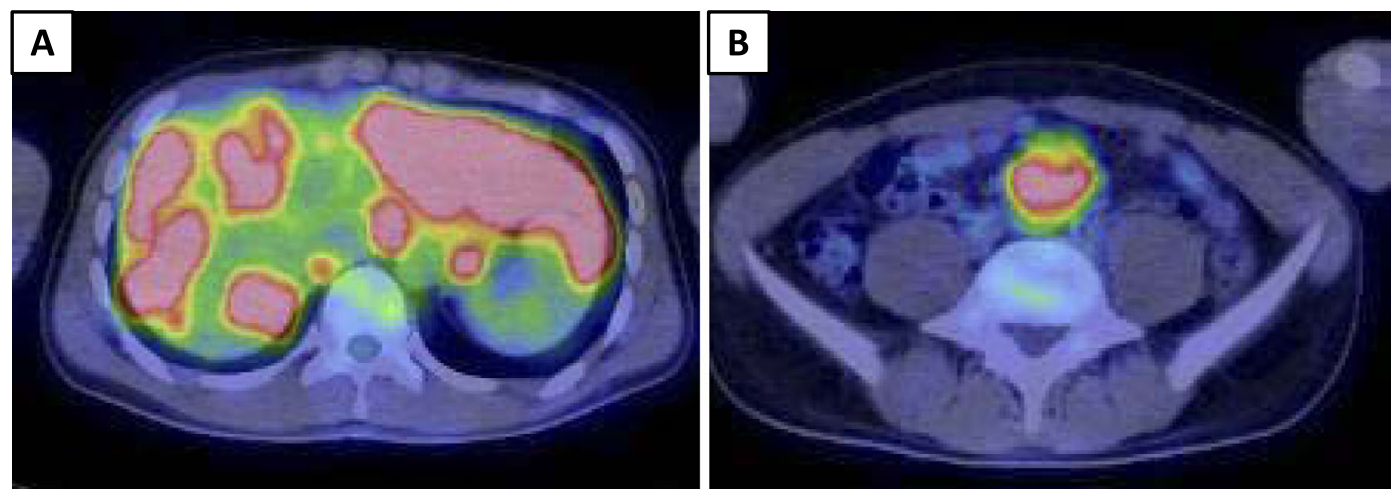

Figure 3. (A) ${ }^{18}$ FDG-PET/CT imaging showed multiple intrahepatic masses exhibiting accumulation of FDG (SUV= 14.6, max). (B) A lower abdominal mass demonstrated accumulation of FDG $(\mathrm{SUV}=6.60)$.

\section{Discussion}

DSRCT is an extremely rare malignancy that was first described by Gerald and Rosai in 1989 (1). DSRCT is predominantly observed in adolescents and young adults. The mean age at diagnosis is approximately 22 years, and the male to female ratio is $4: 1(2,3)$. DSRCT most frequently arises in the peritoneal cavity and may present with peritoneal "caking" of tumor implants (9). Because the tumors can have various differentiations such as epithelial, mesenchymal or neuronal differentiation with coexpression of cytokeratin, EMA, desmin, vimentin and/or NSE, it is difficult to diagnose DSRCT solely based on the immunohistochemical findings $(11,12)$. However, the cytogenetic and molecular genetic analyses of small round cell tumors reveal distinctive findings that help in reaching a diagnosis. The chromosomal translocation $\mathrm{t}(11 ; 22)(\mathrm{p} 13 ; \mathrm{q} 12)$, which leads to fusion of the WT1 and EWS genes, is specific to DSRCT, and the presence of this fusion gene assists with making the final diagnosis $(7,8)$. Making the differential diagnosis is crucial, since treatment strategies differ. The molecular diagnosis of 


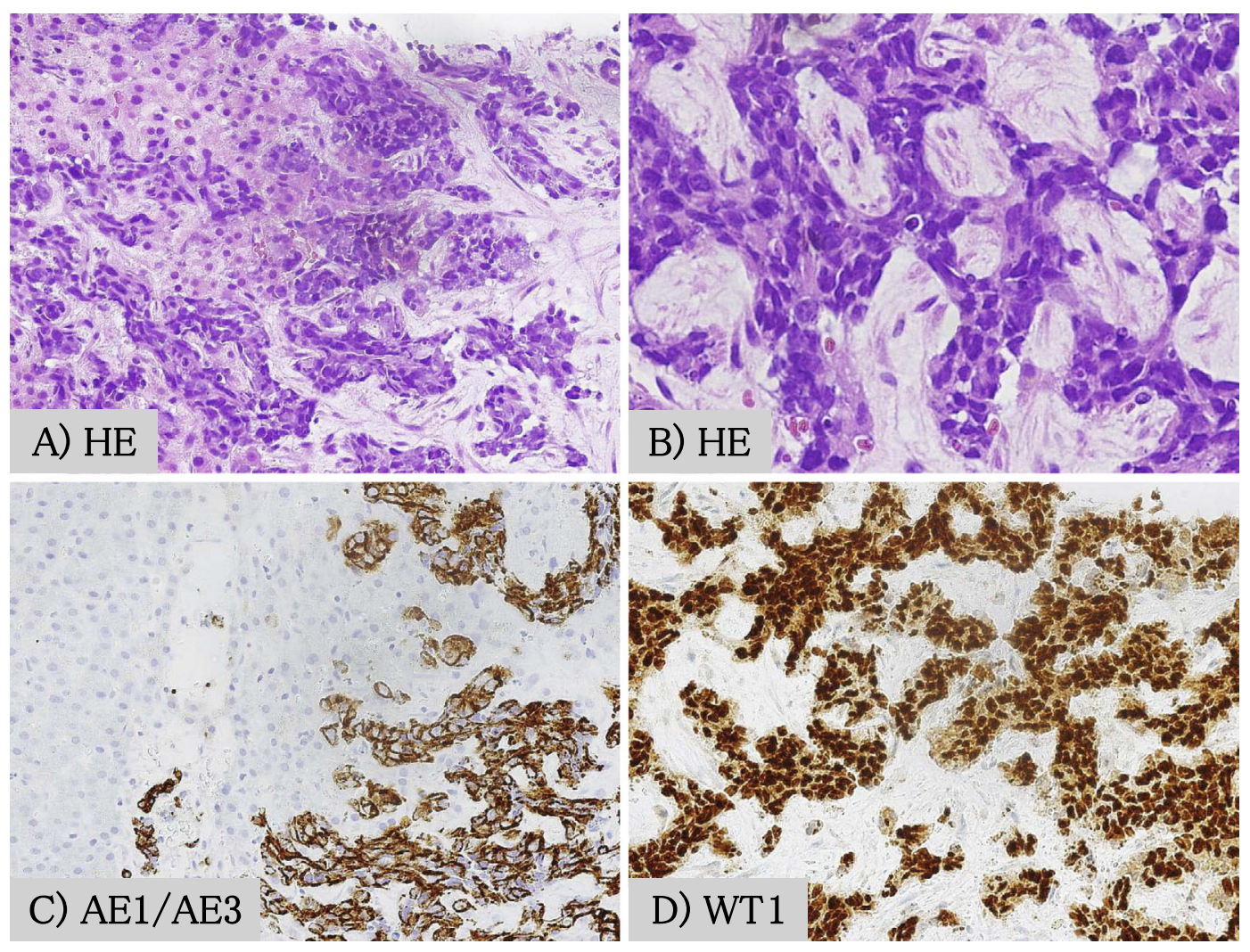

Figure 4. (A) Light micrograph showing compact nests and sheets of small, round cells in fibromyxoid stroma on Hematoxylin and Eosin staining. Magnification 200x. (B) The section exhibited proliferation of atypical cells with hyperchromatic nuclei forming irregularly shaped small nests. (C) AE1/AE3 immunohistochemistry showed typical paranuclear dot-like staining. (D) The tumor cells exhibited a strong nuclear staining reaction for WT1.

\section{A) FISH}

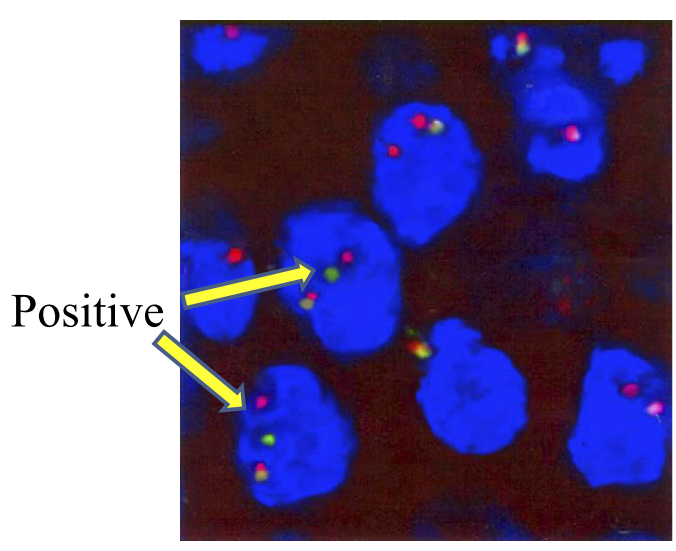

\section{B) RT-PCR}

$\begin{array}{lllllll}\text { MW } & 1 & 2 & 3 & 4 & 5 & 6\end{array}$

400

300

200

100

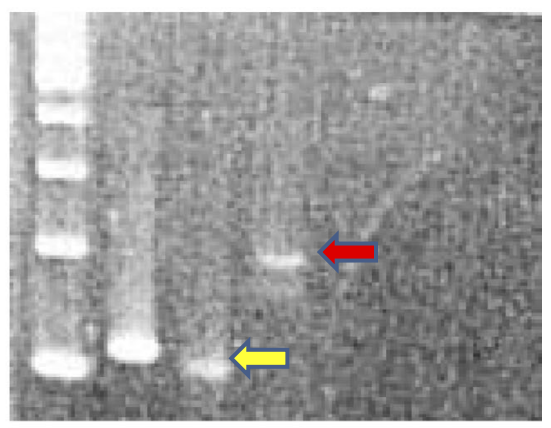

MW (Molecular weight marker)

1 Actin (120 bp)

2 EWS/WT1 exon 8 (103 bp)

3 EWS/WT1 exon 9 (198 bp)

Figure 5. (A) FISH demonstrated tumor cell nuclei with a combined normal red-green signal and a separate red and green signals, indicating the presence of translocation and amplification of the EWS gene (arrow). The red signals are centromeres and the green signals are telomeres. (B) Detection of the EWS-WT1 fusion transcript on RT-PCR revealed a fusion product in EWS with exon 8 of WT1 and exon 9 of WT1. Products ranging from 100 to 200 base pairs (bp) in lanes 1-3, confirming DSRCT, and the absence of amplification in the other lanes. 


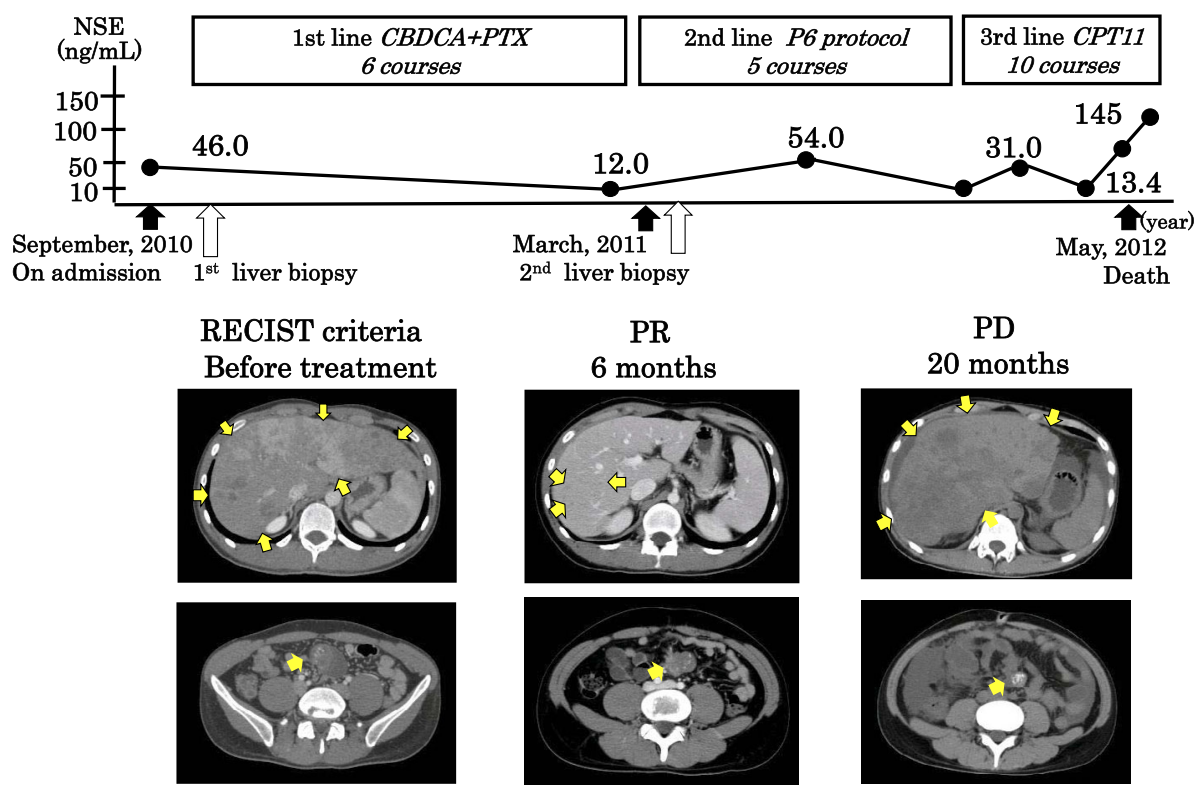

Figure 6. Clinical course of the patient.

Ewing sarcoma/PNET (primitive neuroectodermal tumor) shows the chromosomal translocation $\mathrm{t}(11 ; 22)(\mathrm{q} 24 ; \mathrm{q} 12)$. We can exclude the possibility of Ewing sarcoma/PNET, in this case based on the results of RT-PCR. Therefore, performing biopsies under laparotomy or conducting multiple core biopsies is recommended in order to obtain a sufficient quantity of tumor tissues which in turn can be utilized in relevant examinations. Since we did not obtain adequate tumor samples, we were unable to make a conclusive diagnosis of DSRCT after the initial liver biopsy. We were able to find the EWS-WT1 gene fusion transcript by examining the large sample obtained during the second liver biopsy.

A variety of treatment regimens to treat DSRCT are administered worldwide. Therefore, there is no standard treatment for this disease. Several treatment modalities, including chemotherapy, radiotherapy and debulking surgery, have been used to treat patients with DSRCT (8-12). However, reports regarding these treatments have focused on retrospective data obtained from small numbers of patients. Although DSRCT is known to be rather chemosensitive and radiosensitive, the response is rarely durable (12). There are only a few reports of the role of chemotherapy in such patients. Early treatments involve high-dose systemic polychemotherapy with alkylating agents and the P6 protocol employed at the Memorial Sloan-Kettering Cancer Center. The P6 protocol consists of seven courses of cyclophosphamide, doxorubicin, vincristine, ifosfamide, and etoposide $(2,9)$. Our patient was treated with combination chemotherapy consisting of CBDCA/PTX as first-line chemotherapy, then subsequently treated with the P6 protocol as second-line chemotherapy followed by salvage chemotherapy treatment with irinotecan (CPT-11). Carboplatin and paclitaxel were chosen as the initial regimen of chemotherapy due to the initial diagnosis of carcinoma not otherwise specified (NOS) of unknown primary status, under the NCCN (National
Comprehensive Cancer Network) guidelines. Considerable time had elapsed before the diagnosis of DSRCT was made. The maximum response in this case was a partial remission to the first-line chemotherapy, and the time to progression was six months. The patient was able to manage his ADLs alone with improvement in his abdominal pain associated with the presence of large abdominal masses, even during chemotherapy. Chemotherapy was beneficial for this patient with DSRCT and worth administering. Radiation therapy and volume-reduction surgery are frequently used in patients with advanced DSRCT. However, as the patient's primary prognostic factor was regarded to be metastatic liver cancer and he was not troubled by his lower abdominal mass, we did not opt for these modalities. The prognosis of patients with DSRCT is generally poor. Despite the use of aggressive multimodal treatment, the median survival ranges from 17 to 25 months, with fewer than $20 \%$ of patients achieving 5-year survival (14).

In conclusion, we herein reported the case of a young man who was diagnosed with DSRCT and treated with combination chemotherapy. Molecular genetic studies using FISH and RT-PCR are quite helpful in diagnosing DSRCT. Therefore, the need to obtain a sufficient quantity of tumor tissue for molecular examinations should be emphasized. Our patient responded to polychemotherapy; however, the duration of the response was limited. Further studies are needed to establish a more comprehensive treatment strategy for this refractory disease.

The authors state that they have no Conflict of Interest (COI).

\section{Appendix}

This case study was presented at the 297th Kyushu branch meeting of the Japanese Society of Internal Medicine (May 2012, Fukuoka). 


\section{Acknowledgement}

We would like to thank Dr. Y. Nakashima ${ }^{2}$, Dr. T. Tanaka ${ }^{2}$ and Dr. Aoki ${ }^{3}$ for their support in diagnosing and treating the patient $\left({ }^{2}\right.$ Department of Medical Oncology, Hematology and Infectious Disease, ${ }^{3}$ Department of Pathology, Fukuoka University Faculty of Medicine).

\section{References}

1. Gerald WL, Rosai J. Desmoplastic small cell tumor with divergent differentiation. Pediatr Pathol 9: 177-183, 1989.

2. La Quaglia MP, Brennan MF. The clinical approach to desmoplastic small round cell tumor. Surg Oncol 9: 77-81, 2000.

3. Gerald WL, Miller HK, Battifora H, Miettinen M, Silva EG, Rosai J. Intra-abdominal desmoplastic small round-cell tumor. Report of 19 cases of a distinctive type of high-grade polyphenotypic malignacy affecting young individuals. Am J Surg Pathol 15: 499-513, 1991.

4. Basade MM, Vege DS, Nair CN. Intra-abdominal desmoplastic small round cell tumor in children: a clinicopathologic study. Pediatr Hematol Oncol 13: 95-99, 1996.

5. Norton J, Monaghan P, Carter RL. Intra-abdominal desmoplastic small cell tumor with divergent differentiation. Histopathology 19: 560-562, 1991.

6. Ordöñez NG. Desmoplastic small round cell tumor: II: an ultrastructural and immunohistochemical study with emphasis on new immunohistochemical markers. Am J Surg Pathol 22: 1314-1327, 1998.

7. Rodriguez E, Sreekantaiah C, Gerald W, et al. A recurring translocation, $\mathrm{t}(11 ; 22)(\mathrm{p} 13 ; \mathrm{q} 11.2)$, characterizes intra-abdominal desmoplastic small round-cell tumors. Cancer Genet Cytogenet 69: 1721, 1993.

8. Sawyer JR, Tryka AF, Lewis JM. A novel reciprocal chromosome translocation $\mathrm{t}(11 ; 22)(\mathrm{p} 13 ; \mathrm{q} 12)$ in an intraabdominal desmoplastic small round-cell tumor. Am J Surg Pathol 16: 411-416, 1992.

9. Kushner BH, LaQuaglia MP, Wollner N, et al. Desmoplastic small round-cell tumor: prolonged progression-free survival with aggressive multimodality therapy. J Clin Oncol 14: 1526-1531, 1996.

10. Leuschner I, Radig K, Harms D. Desmoplastic small roundcell tumor. Semin Diagn Pathol 13: 204-212, 1996.

11. Kretschmar CS, Colbach C, Bhan I, Crombleholme TM. Desmoplastic small round cell tumor: a report of three cases and review of the literature. J Pediatr Hematol Oncol 18: 293-298, 1996.

12. Lae ME, Roche PC, Jin L, et al. Desmoplastic small round cell tumor: a clinicopathologic, immunohistochemical, and molecular study of 32 tumors. Am J Surg Pathol 26: 823-835, 2002.

13. Therasse P, Arbuck SG, Eisenhauer EA, et al. New guidelines to evaluate the response to treatment in solid tumors. J Natl Cancer Inst 92: 205-216, 2000.

14. Armelle D, Philippe C, Laure C, et al. Desmoplastic small round cell tumor: current management and recent findings. Sarcoma 2012: 714986, 2012.

(C) 2013 The Japanese Society of Internal Medicine http://www.naika.or.jp/imonline/index.html 